\title{
Robotic Orthosis for Upper Limb Rehabilitation
}

\author{
Fernanda Márcia Rodrigues M. Ferreira 1,; Guilherme de Paula Rúbio; Fabrício Henrique de \\ Lisboa Brandão ${ }^{2}$; Arthur Mazzini da Mata²; Natália Batista Castilho de Avellar³; João Paulo \\ Fernandes Bonfim ; Leandro Gonzaga Tonelli 5; Thales Gomes Silva6; Rina Mariane Alves \\ Dutra $^{1,6}$; Adriana Maria Valladão Novais Van Petten ${ }^{3}$; Claysson Bruno Santos Vimieiro ${ }^{1,2,7}$ \\ 1 Graduate Program in Mechanical Engineering, Universidade Federal de Minas Gerais, Belo Horizonte, \\ MG, Brazil; guilhermeprubio@gmail.com; claysson@ufmg.br \\ 2 Department of Mechanical Engineering, Universidade Federal de Minas Gerais, Belo Horizonte, MG, \\ Brazil; fabriciohlisboa@ufmg.br; arthurmazzini@ufmg.br \\ 3 Department of Occupational Therapy, Universidade Federal de Minas Gerais, Belo Horizonte, MG, Brazil; \\ nataliabcavellar@gmail.com; avaladao@ufmg.br \\ 4 Department of Electrical engineering, Universidade Federal de Minas Gerais, Belo Horizonte, MG, Brazil; \\ jpfb2015@ufmg.br \\ 5 Laboratório de Bioengenharia-LABBIO, Universidade Federal de Minas Gerais, Belo Horizonte, MG, \\ Brazil; leandrotonelli@ufmg.br \\ 6 Centre for Innovation, Research and Teaching in Mechatronics, Universidade Federal de São João del-Rei, \\ Ouro Branco, MG, Brazil; thalesgomes28@gmail.com; rina@ufsj.edu.br \\ 7 Graduate Program in Mechanical Engineering, Pontifícia Universidade Católica de Minas Gerais, Belo \\ Horizonte, MG, Brazil; \\ * Correspondence: fernandaferreira.to@gmail.com; Tel.: +33-07- 6689-1257
}

\begin{abstract}
Individuals with impaired upper limb have motor limitations that interfere with functionality. An alternative to rehabilitation consists of Robot Assisted Therapy, a method that increases the effectiveness of treatment. New robotic actuators have been developed to assist in the rehabilitation of the upper limb. One of them aims to actively perform finger extension and flexion passively, using a servo motor coupled to a rope system. At the elbow, a DC motor combined with a gearbox was coupled to a system of pulleys and ropes designed to actively perform flexion and extension movements. To activate the system, an Arduino-NANO® and a mobile application for Android were used. The performance of the prototype was evaluated in four post-stroke volunteers. The ability to perform the proposed movements with the device was observed. Structural reinforcement was necessary, after twisting the elbow support structure, with pronation of the forearm, resulting in increased component weight. This work presented new robotic devices that can assist in the rehabilitation of post-stroke individuals.
\end{abstract}

Keywords: Robot Assisted Therapy, Stroke, Rehabilitation. Assistive Technology.

\section{Introduction}

Stroke consists of a clinical syndrome, with vascular origin, which presents a reduction in the blood supply to brain structures, characterized by rapid development of focal or global signs of brain function disturbance [1], is being one of the main causes of death and disability acquired in adults worldwide [2,3]. Upper extremity (UE) impairment is the most common disabling deficit after stroke $[4,5]$ is seen in approximately $70 \%$ of individuals [1,6]. Kwakkel et al. [7] show that about 30 to $66 \%$ of patients do not have upper limb function on the affected side, when evaluated 6 months after the stroke and, despite intense rehabilitation efforts, only $5 \%$ to $20 \%$ demonstrate complete functional recovery. Robot-assisted Therapy (RT) is an emerging and innovative form of intervention that [8]offers training in dosage and much higher intensity [9], in an interactive, individualized, adaptable 
and quantifiable way [10-13], thus, improving the strategies of relearning motor and functional results.

Several systematic reviews have been carried out to investigate the effects of RT in post-stroke individuals [7,12,14-20]. BERTANI et al. [19] and ZHANG et al. [18] found that in individuals post chronic stroke, robotic therapy was more effective in reducing motor impairment than conventional therapy, but not in patients with acute stroke. The most recent systematic review with meta-analysis carried out by Mehrholz et al. [20] concluded that robot-assisted arm training can improve activities of daily living, arm function and muscle strength with a high quality of evidence. However, the authors state that the results should be interpreted with caution, as there were variations between the tests in intensity, duration, and amount of training; type of treatment; characteristics of participants and outcome measures used.

Despite all the benefits of robotic therapy, most of the robotic orthoses for upper limb rehabilitation currently have numerous disadvantages, limiting their application and restricting their use on a large scale. They have a high cost, material, and unfavorable aesthetics (they are robust, bulky, and heavy), making portability impossible or difficult, interfering with the ability to perform activities of daily living. Therefore, it is necessary to develop new studies, focusing on more effective rehabilitation equipment, with lower cost, better functional results, aesthetically pleasing with high technological performance, aimed at the needs of people with disabilities.

Thus, this study aims to develop a robotic orthosis for individuals with motor impairment of the upper limb resulting from a stroke that helps flexion and extension movements of the elbow and fingers and validate the device in volunteers.

\section{Experiments}

\subsection{The Device}

The equipment is characterized by its modularity, composed of two independent modules, being the hand module and the elbow module, both can act together or separately. Next, the orthosis will be detailed, divided into two parts: static orthosis and mechanical structure, the first consisting of parts that attach to the user's arm and position it, and the last one is made up of the other parts. Finally, the control system and the actuators motors will also detail. The Figure 1 has shown a orthoses schematic view.

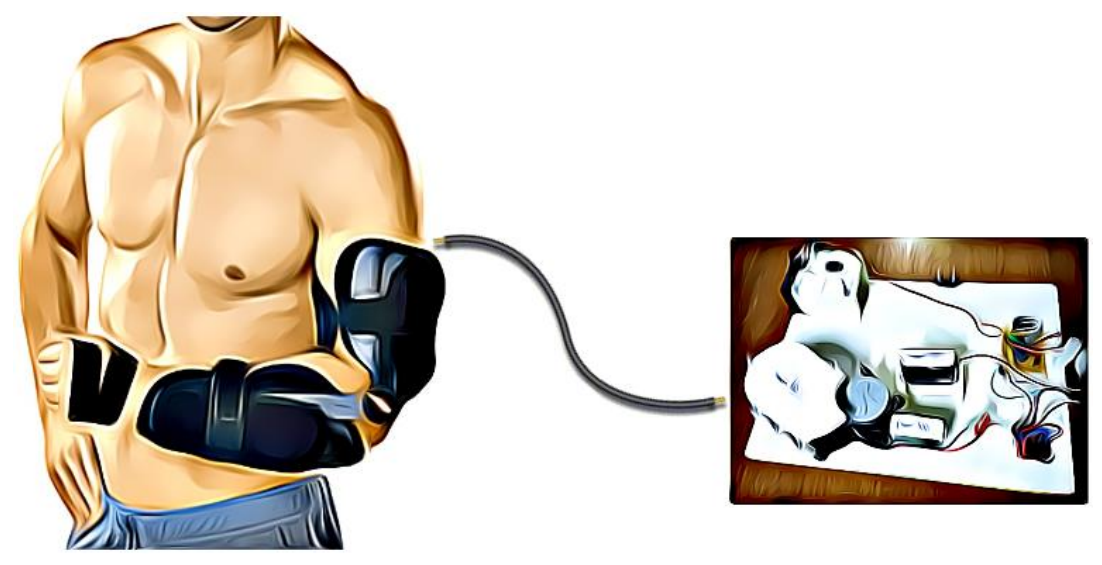

Figure 1. Schematic representation of the device developed.

\subsubsection{Hand module-Mechanical Design}

The hand module actuation mechanism performs the flexion and extension movement of the fingers and was make up of artificial tendons, artificial phalanges, artificial metacarpal, and fingerstall. For the artificial tendons, Kevlar multi-wire cables were used, because it is light, durable, flexible, has a low friction coefficient, tensile strength, low cost, and low percentage elongation. These advantages were evidenced in a previous work, developed by these authors [21], using the EMIC 23$5 \mathrm{D}$ machine for the tensile test, was being possible to generate the rope deformation graph using the 
Tesc Software version 3.04 and the test method is called rectangular tie pull. The artificial phalanges consist of rigid lactic polyacid (PLA) rods, it has a function of limiting the movement of the user's phalanges, preventing hyperextension of the metacarpophalangeal joints and flexion of the interphalangeal joints during a finger extension. They are attached to the fingerstall and have a hole that passes each of the artificial tendons, used to extend the patient's fingers when these tendons are pulled, as seen in the Figure 2.

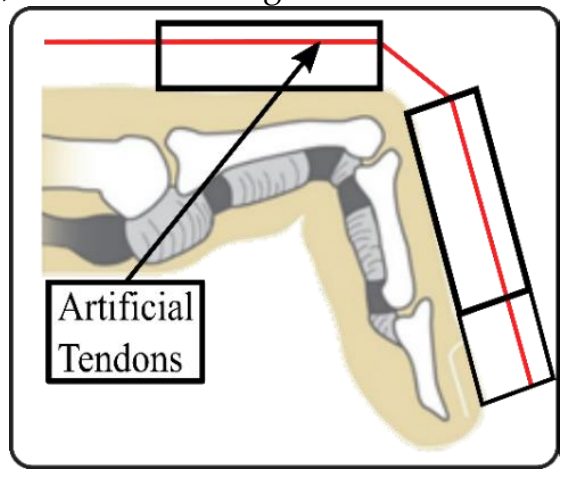

(a)

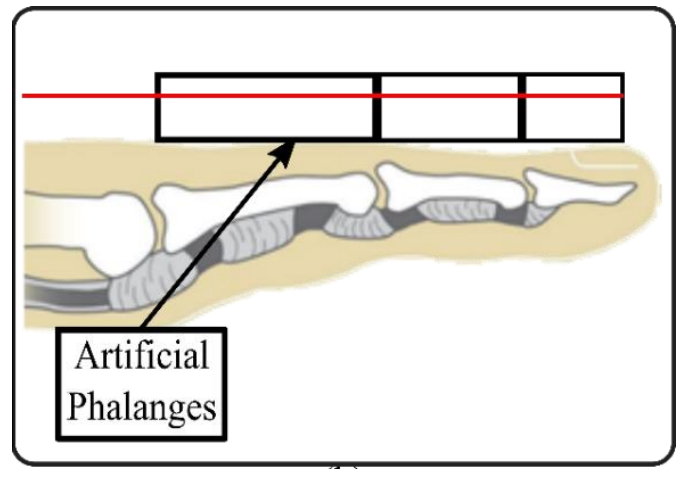

(b)

Figure 2. Schematic mechanism representation of (a) closing and (b) opening fingers with artificial phalanx and tendons. Adapted from Rúbio et al. [22]

To the mechanism perform the correct movement a cable coming from the external motor was connected in an artificial metacarpal using conduits. The artificial metacarpal was responsible to support and connection of that cable with the four artificial tendons use in the fingers. The artificial tendons passed through the artificial phalanges and were also guided through smaller holes to the larger hole in the center of the artificial metacarpal. All tendons were joined to the cable by a plastic piece, specially designed to join and adjust the length of all artificial tendons. In this way, when the external motor tensioned the cable, that tension was transmitted to the artificial tendons, each extending one of the fingers as requested.

In order to perform the flexion movement of the fingers, the artificial tendons are gradually relaxed and the fingers passively return to their original position, due to the elevated spasticity present in the superficial flexor muscles of the post-stroke user fingers.

\subsubsection{Hand module-Static Structure}

The hand module static orthosis was composed by rigid splints made of thermoplastic in the ventral and dorsal forearm, and pieces made of Neoprene which casing the splints of the arm and forearm and the fingers. In the hand, rigid splints have the function of positioning correctly the limb and contain hypertonia. The positioning of the wrist and thumb is performed through the ventral splint, which covers the ventral part of the forearm, extending through the wrist and surrounding the thumb, ending at the base of the palm. The wrist should be positioned at $20^{\circ}$ of extension and $14^{\circ}$ of ulnar deviation and thumb in abduction, providing the functional position [23] and the ability to successfully perform daily activities.

An artificial metacarpal was design to joining the artificial tendons from each finger with an acting rope. Thus, when the actuation system tensioned the action rope, the artificial tendons was pulled, and the fingers extension was performed as previously explained. Others functions of the artificial metacarpal were of bulkhead, preventing hyperextension of the metacarpophalangeal joints and structural support for the rope conduit. A complete viewing about the hand module is shown in Figure 3.

A long piece that sits on the patient's forearm and made of PLA called a Linear Rail was used to house the elbow rod and let it slide freely, adding a second degree of freedom to the elbow axis since 
it was observed that during the movement of the elbow its axis changes slightly. The main function of the Linear Rail was to connect the hand module to the elbow module.

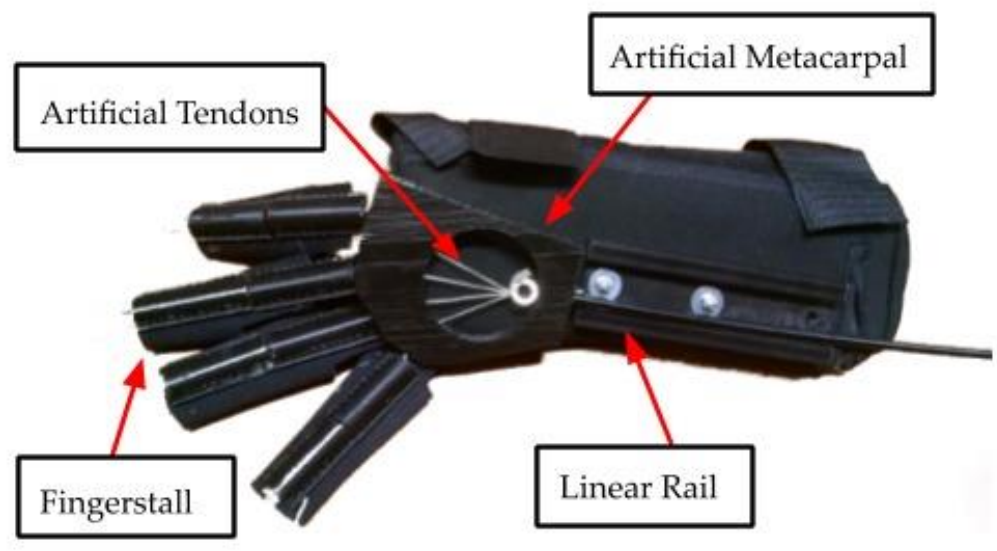

Figure 3. Hand module

\subsubsection{Elbow Module- Static Structure}

The elbow module static structure was composing by an arm splint and a shoulder pad. The arm splint had the function of supporting the mechanical parts of the actuators and covers the external part of the arm with an opening in top, due to the fact that the triceps slightly change its volume in relation to the biceps during the elbow extension and flexion.

The shoulder pad was a strip of Neoprene that trough over the shoulder and diagonally wrapped the user's torso. The ends of the band were on the side of the abdomen, where a Velcro joins them. On the shoulder, three Velcro straps fasten the shoulder to the casing of the arm. The shoulder pad fastens the orthosis preventing it from slipping and allowed weight distribution across the trunk.

\subsubsection{Elbow Module-Mechanical Design}

The actuation mechanism of the elbow module consists of a lever system designed through a rod coupled to a pulley, which when performing the rotation movement extends and flexes the user's elbow. The rod consists of a shaped plate, connected to a transmission pulley that connects the casing of the arm to the forearm performing the rotation movement. Beyond the rod with pulley a pulley base was designed. That base was a part manufactured through 3D printing, using PLA filament, and was coupled to the arm's thermoplastic splint using four steel screws for fixation. Two conduits come from the external motors and has connected at the base of the pulley. These conduits were fastened with a cover (also made in 3D printing) using steel screws. Inside each conduit, there was a rope whose other end was attached to the pulley of the rod, so that when one of these ropes was tensioned, the other was released, and vice versa, performing the flexion and extension movement of the patient's arm.

During the test with one volunteer, it was observed that the elbow extension and flexion, a twisting movement occurred at the pulley base that prevented the rod movement. In addition, it is possible to observe a pronation movement of the volunteers' arm, and that was a cause of the twisting in the pulley base, evidenced by the distance between the pulley and the volunteer's elbow joint, shown in Figure 4. Thus, it was necessary to increase the robustness of that part, to support the loads and prevent the user's pronation. movement. 


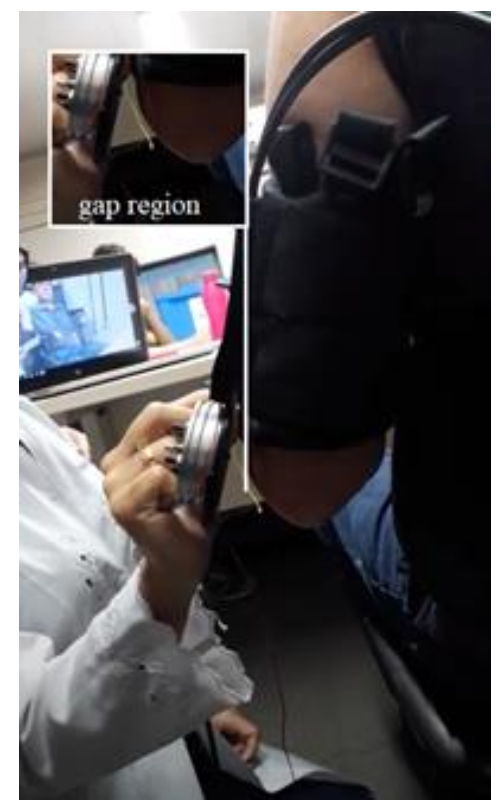

Figure 4. The pulley base twisting.

After all the reinforcements in the pulley base the movements has been performed with and the twisting was not observed. The elbow module full view was shown in Figure 5.

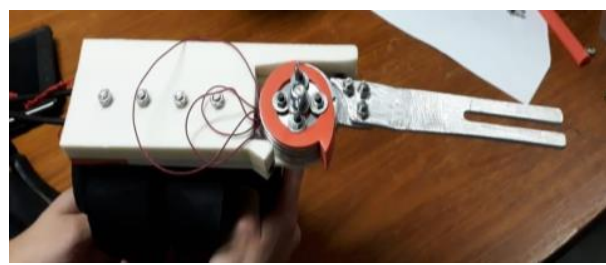

Figure 5. The Elbow module.

\subsubsection{Motors and Control}

The motors were external to the orthosis, the movement of the motors was transmitted through ropes that connect the motor to the actuation system present in the orthosis. The ropes were routed to the mechanism via conduits. For the elbow module, a pulley system was used two ropes and gear box to multiply the torque and decrease the speed. The pulley was attached to the arm rod. One rope moved the pulley clockwise and the other counterclockwise. To perform the elbow extension and flexion movement, one of the ropes was tensioned, causing the pulley to be moved and pulled the other cable.

To drive the elbow module, a DC motor from the manufacturer MABUSHI was selected, with $12 \mathrm{~V}$ of nominal voltage and a nominal torque of 1 N.m. The engine was selected due to its availability on the market, usability, and low cost. Beyond the motor was necessary determine the loads generated by the elbow extension and flexion movement, to design a reduction system necessary. For that was necessary to build a dynamic model to determine the minimum torque required to move it at a constant speed.

In the dynamic model only a weight of the hand module and forearm of de user was used like loads in the system. In that way, was used the anthropometric values of weight and length of the $\mathrm{limb}$ to locate the system center of gravity (CG) and calculate the necessary torque ( $\mathrm{T}_{\mathrm{o}}$ ). To meet the system dynamic was considered a constant angular velocity of movement to $10^{\circ}$ up to $120^{\circ}$ of elbow position. Using those characteristics using the Equation (1), where $\mathrm{R}_{\mathrm{g}}$ was the distance between the elbow joint center and CG of the set, $\mathrm{P}$ was the weight force and $\theta$ was the elbow joint angle determined through cinematics relationships in constants angular velocities. 


$$
\mathrm{T}_{\mathrm{o}}=\mathrm{P} \cdot \mathrm{R}_{\mathrm{g}} \cdot \sin \theta,
$$

Using the software Matlab, the torque variation was then calculated needed over time, as shown in Figure 6. To develop that graphic was used a $\mathrm{Rg}$ value of 195,20 $\mathrm{mm}$ (obtained using an anthropometric values), a weight of $2.5 \mathrm{~kg}$ of the system and an angular velocity of $22 \mathrm{rad} / \mathrm{seg}$.

Required Torque $\mathbf{x}$ Time

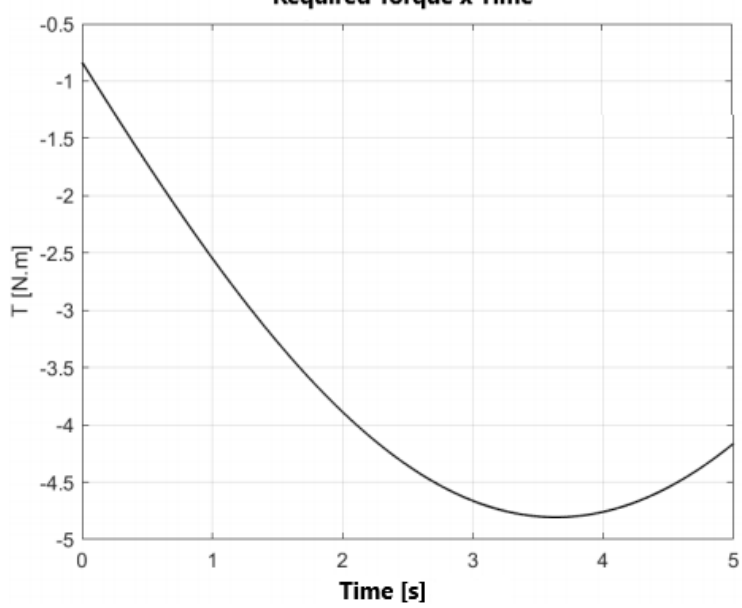

Figure 6. Variation of the required torque to flex the elbow over time.

How could be observed in Figure 6 the estimated peak torque was $4.8 \mathrm{Nm}$, however your value change during the movement. Because of this, to ensure the correct movement a gear box with a ratio of 12:1 was design, in that way the coupled system motor and gear box had an output torque about $12 \mathrm{Nm}, 2.5$ times higher than the necessary torque give safety of the system.

For the hand module, a servo motor of model HPI SFL-10 MG WATER RESISTANT SERVO, with $6 \mathrm{~V}$ of nominal voltage, and steel gears were selected, which gives a leaner reduction system and a nominal torque of $2.35 \mathrm{Nm}$.

The motors were driven by limit switches to indicate the end of the movement. For the processing of the commands, a firmware was developed and recorded in an Arduino-NANO ${ }^{\circ}$ microcontroller. The firmware used interaction with a mobile application developed by Labbio for Android platforms, named as Órtese. This application has a command menu where it is possible to select the module to be activated and perform manual or automatic tasks. Parameters such as speed, number of repetitions, and range of motion can be defined by the user. The Figure 7 shows some of the main screens of the application.

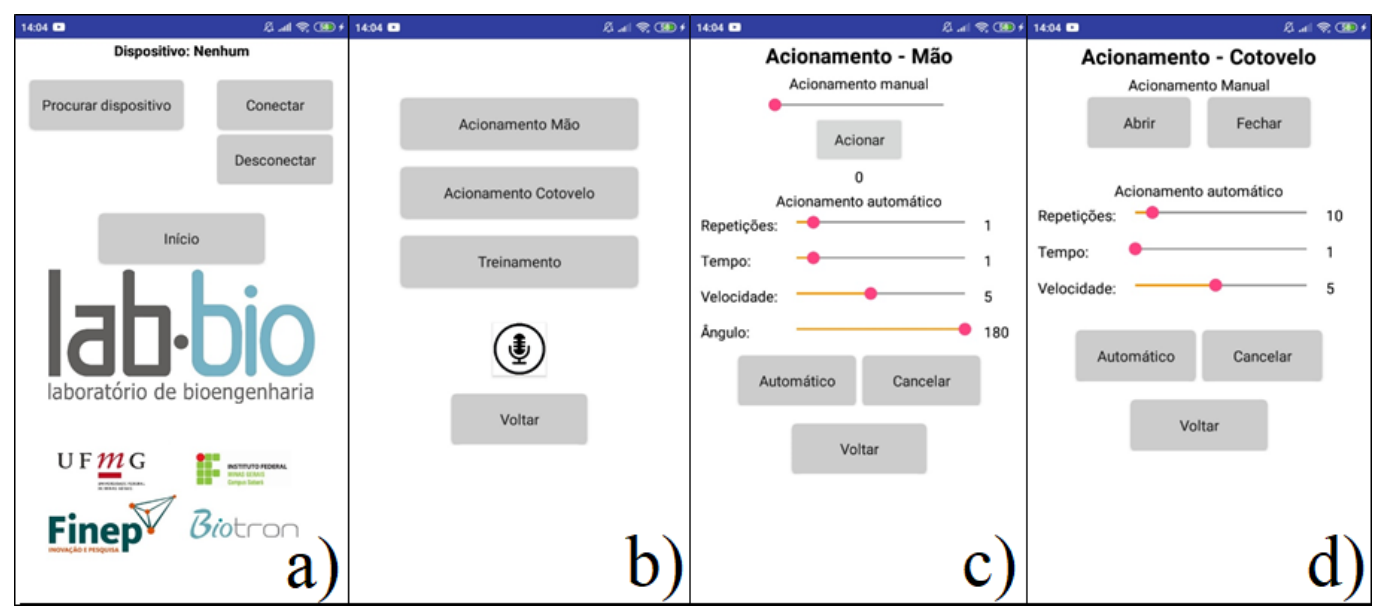

Figure 7. Home screen (a), main menu(b), hand module control (c), elbow module control (d). 
The commands of the mobile application are sent to the microcontroller through the Bluetooth module HC-05, the microcontroller also sends a PWM signal to an $\mathrm{H}$ bridge to control the motors. The Figure 8 shows the motors and electronics responsible for the control.

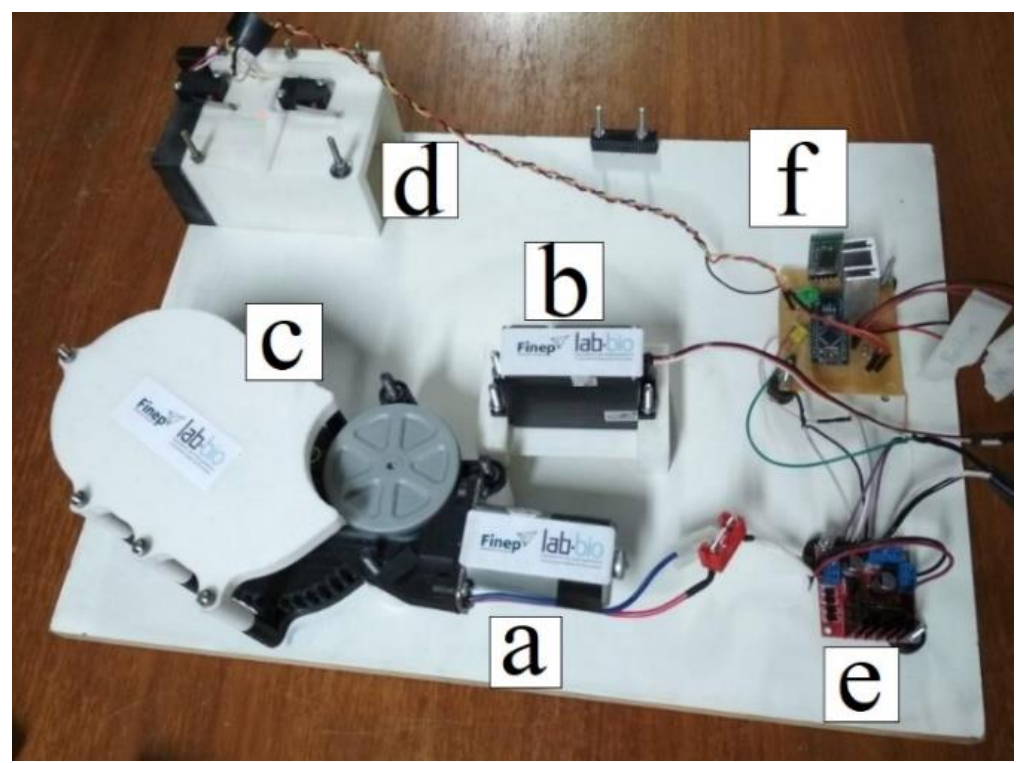

Figure 8. DC motor (a), servomotor (b), reduction gearbox (c), limit switches (d), DC Motor H bridge (e) and controller (f).

\subsection{Clinical test}

The prototype was validated in its biomechanical aspects, structure, interface, components, safety, and usability. The biomechanical functioning and the need for changes and adaptations in the device were verified, as well as the adaptation to the needs of each patient and injury, privileging comfort, functionality, and usability.

\subsubsection{Volunteers}

Study participants were recruited through stroke associations, rehabilitation centers, hospitals, and social media. Participants were selected according to the inclusion and exclusion criteria and informed about the objectives of the study. After the participants' consent, they signed the Free and Informed Consent Form (ICF). The study project was approved by the Research Ethics Committee (COEP) of the Federal University of Minas Gerais (CAAE Registry: 22207213.5.0000.5149).

The inclusion criteria in the study were: individuals aged 18 years or older with a diagnosis of stroke in the chronic phase (evolution time above six months) [24]; hemiparetic, with reduced motor function in the upper limb, alteration in tone from mild to moderate (Modified Ashworth Scale <3) [25]; without impairment of sensitivity assessed using Fulg Meyer [26] and without severe cognitive deficits, assessed using the Brazilian version of the Mini-Mental State Examination (the cutoff for illiterate individuals 13, 18 for low and medium schooling and 26 for high schooling [27]. Were excluded individuals with a complete return of upper limb motor function; bilateral motor impairment; severe additional orthopedic or rheumatological impairment, before stroke; severe pain in the affected upper limb, measured using the Visual Analogue Scale (VAS) ( $>8$ on a scale of 0 to 10); open lesions on the skin where the device will be attached; use in the last three months of botulinum toxin for spasticity or other drugs known to increase recovery motor, as well as participation in the last 3 months of another research study to improve upper limb function.

Four participants were selected for the study and the average age of the participants was $46 \pm$ 17.59 years, $50 \%$ were men and the average post-stroke time was $19.83 \pm 7.17$ months. The characteristics are shown in Table 1.

Table 1. Participants characteristics 


\begin{tabular}{ccccccc}
\hline \multirow{2}{*}{ Participants } & \multirow{2}{*}{ Sex } & Age & Dominance & Post stroke time & \multicolumn{2}{c}{ Spasticity Level } \\
& & & & & Fingers & Elbow \\
\hline 1 & Male & 73 & Right & 53 months & 1 & 1 \\
2 & Male & 38 & Right & 113 months & 2 & 2 \\
3 & Female & 25 & Left & 48 months & 2 & 2 \\
4 & Female & 48 & Right & 24 months & 1 & $1+$ \\
\hline
\end{tabular}

\subsubsection{Procedures}

The study was carried out on the premises of the Bioengineering Laboratory (LABBIO) of the Mechanical Engineering Department of the School of Engineering and in the Assistive Technology Laboratory of the Occupational Therapy Department of the School of Physical Education, Physiotherapy and Occupational Therapy, both at the Federal University of Minas Gerais (UFMG). The tests with the orthosis were performed by the responsible researcher (occupational therapist) and monitored by students from the areas of engineering, physics, and physiotherapy

Participants sat on a chair in front of a height-adjustable table. The orthosis was fixed on his upper limb, who remained with the device for one hour, and anatomical adjustments, possible pressure points on the skin, participants' posture, as well as safety and comfort were checked. The ability to perform repetitions of the elbow and finger flexion and extension movements, individually and simultaneously, was analyzed, as well as the gripping of different objects, maintaining their fixation during the movement of these objects. The orthosis was controlled via an application, developed by the group of researchers, under the guidance of a health professional.

The tests happened for one month and they were accomplished weekly in one-hour sessions. They were filmed to further analysis from the group of researchers and the exact identification of the main issues to be modified. Despite the video analysis, the participants' feedback, with questions during the use of the device, was essential in this stage.

\section{Results}

After the construction of the reduction system, the bench tests were redone by repeating all the conditions of the previous tests. The orthosis was tested in these four volunteers, all affected by stroke. The first test was carried out with the activation of the hand module. In this test was solicited the volunteers' fingers opening of $0^{\circ}$ to $180^{\circ}$, thus simulating a full opening fingers movement. The orthosis was able to open the volunteer's hand, as shown in Figure 9.

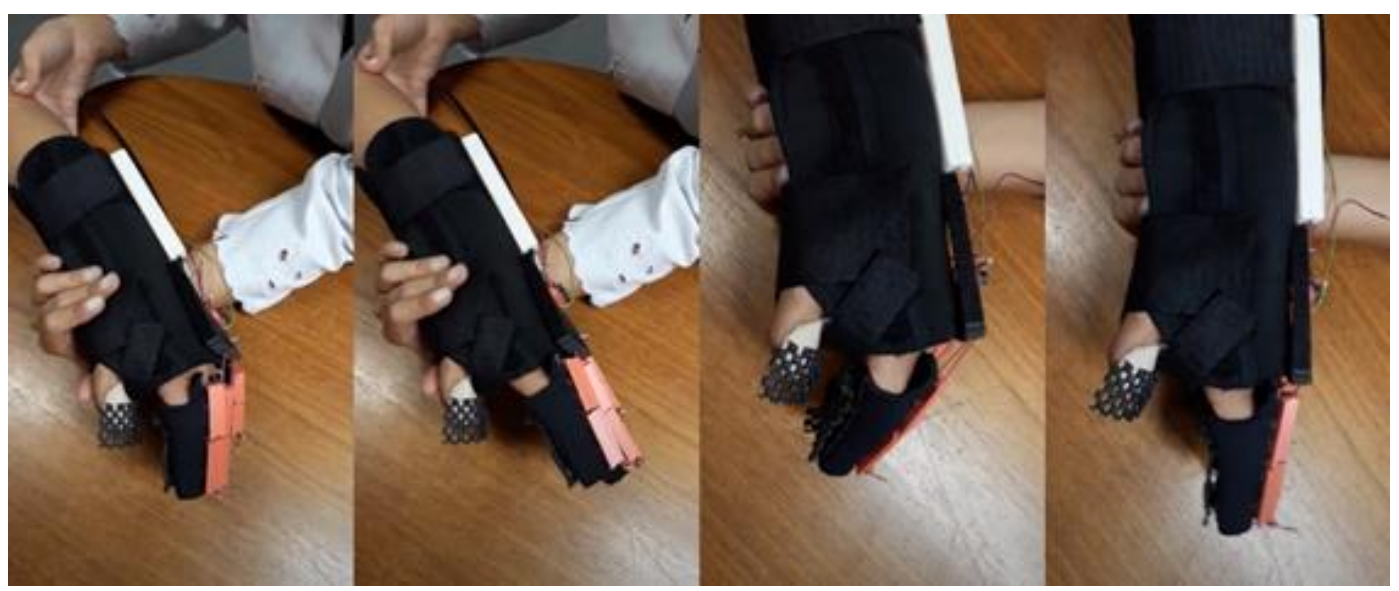

Figure 9. Hand opening tests..

After performing the hand module test, the elbow module was tested. The elbow actuator was setup to make a full flexion and extension, as shown in Figure 10. How can be observed, the movement intended was performed how desired. 


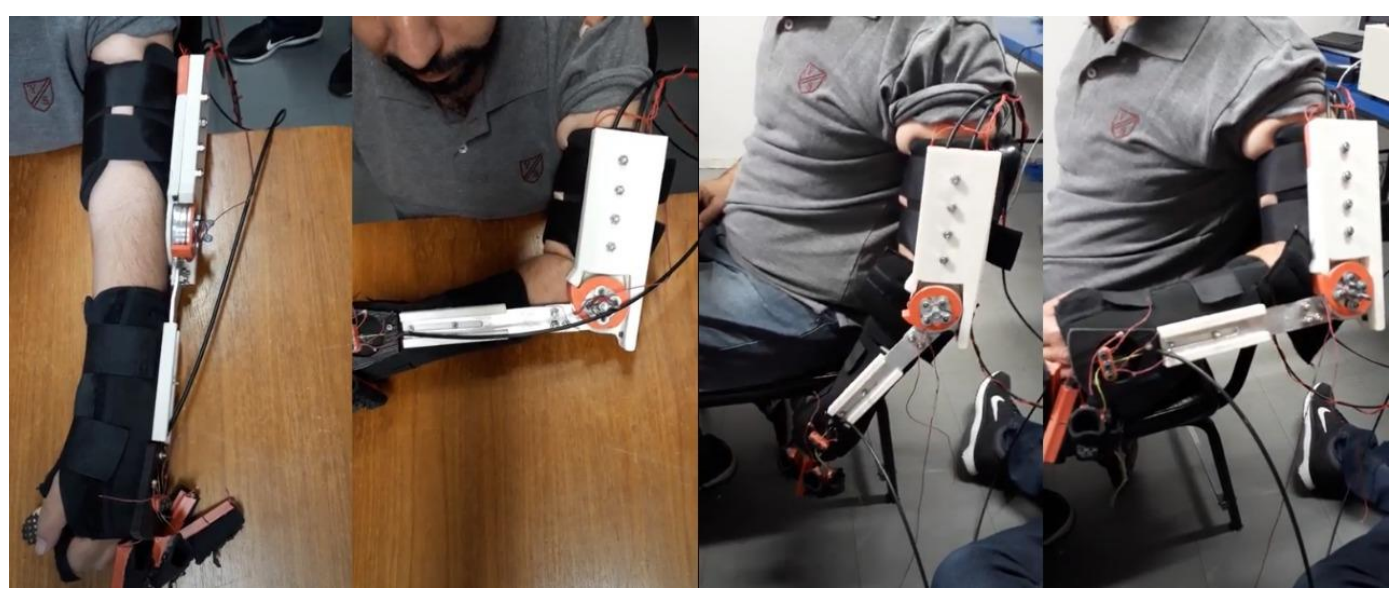

Figure 10. Elbow movement tests.

The elbow module presented difficulties to perform the movement of closing the elbow of the volunteers and it was necessary to use the therapist's support for the movement to be completely performed. During the tests with a volunteer, when trying to perform the movement without the support of the therapist, the main gear of the transmission system fails and needed to be replaced, and the task can be performed correctly. No damage to the engines or ropes was observed.

After validating the correct operation of the device, a test was performed involving holding objects tasks. Figure 11 shows the task performed for a bottle and Figure 12 shows the task performed for a ball.

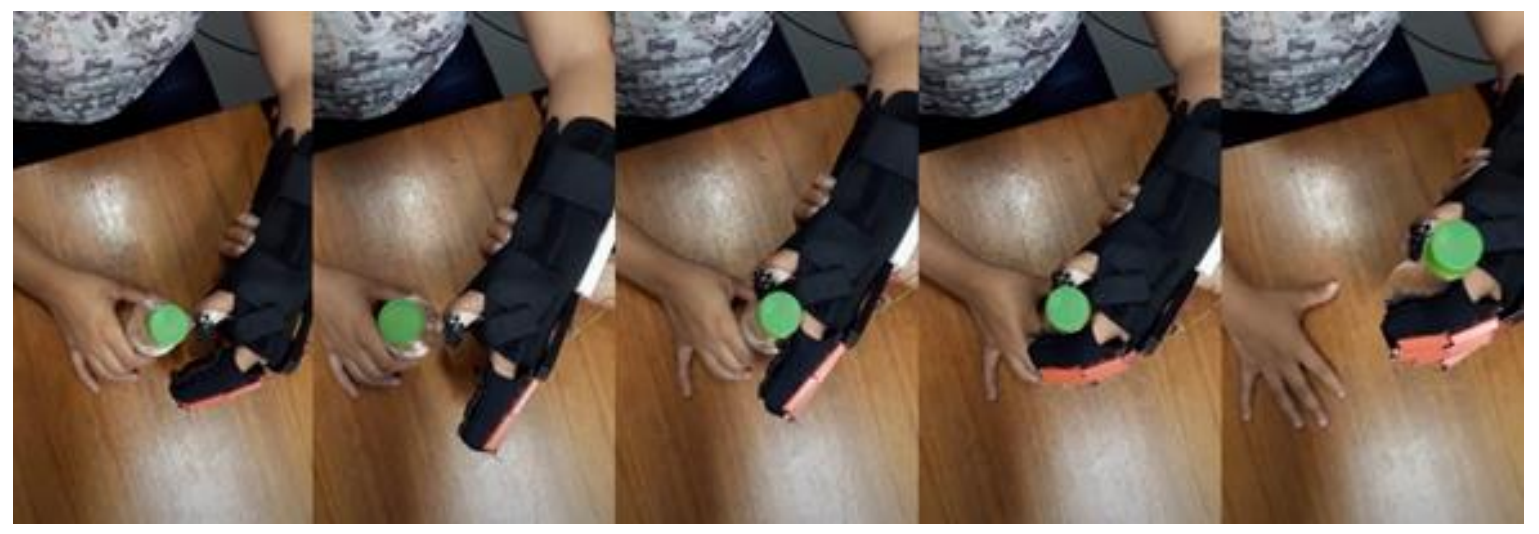

Figure 11. Grabbing a water bottle task.

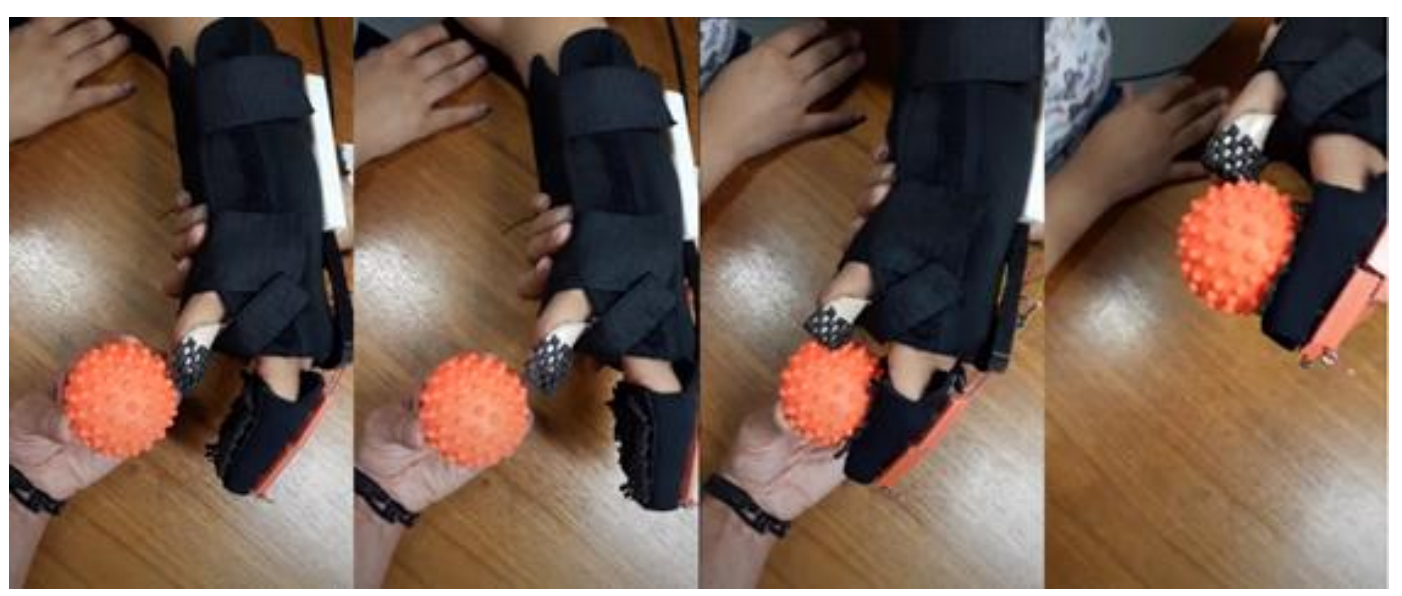

Figure 12. Grabbing a ball task. 
The orthosis was able to assist the volunteers to carry out the task of picking up fixed objects and maintained their fixation during the movement of these objects.

During the tests, correct positioning of the limb and proper posture were observed, and pressure points on the skin have not been identified. The participants did not report discomfort, tingling and paresthesia when using the device, however, high weight was reported.

\section{Discussion}

Clinical tests showed that the equipment was able to perform the movements effectively, with correct biomechanical functioning. However, difficulties and limitations were observed during the development of the device, since the fingers interfered with each other and the elbow module presented relative difficulty in performing the volunteers' elbow closing movement.

Islam et al. [28] e Gull et al. [29] mention the difficulty in the development of robotic exoskeletons due to the anatomical structure of the human upper limb is very complex and flexible, with high number of joints, muscles and planes of movement [28-30]. We observed this complexity in the elbow joints, where the instantaneous centers of rotation (ICR) change it with the joint movement [28]. The axis of rotation of the flexion and extension of the human elbow is slightly inclined - medial-lateral (and not perpendicular) to the frontal plane of the arm, as normally occurs in other joints. As a result, their relative position is not fixed during the movement, varying as the elbow flexes or extends [31]. Besides that, in addition to the elbow joint making a predominant uniplanar movement of flexion and extension, it also performs an accessory movement, with a small axial rotation (that is, rotation around its longitudinal axis) and movement from side to side as it flexes and extends) [31]. These phenomena interfere in the development of robotic applications to the upper limb and according to Neumann [31] they must be taken into account by $t$ by bioengineers when designing elbow orthoses.

In a way to overcome those complexity, the rail developed give one more degree of freedom to the elbow actuator, this way compensates the ICR anatomical changes. Like shown in Figure 10, using the device the volunteer could perform the elbow extension and flexion. During the movement, the rod inside the rail slides, in the way to adapt with the anatomical characteristic of the user and made the movement. However, in the clinical test all users claimed the excessive weight of the elbow module. To continue the device evaluation, the elbow module was tested only over a desk, in that way, the user was not affected by the excessive weight. Due to the actuator unilateral structure your CG was located external to the arm and far of the limb center line, because of this the user's sensation weight was elevated. The change in the CG location must be made, one way where the device CG was near to the arm center. We propose change the unilateral structure for a bilateral structure making the device light and able to use without problems to the users.

We also observed this complexity in the wrist, hand, and fingers segments, with numerous joints, muscles, and planes of movement. However, one alternative to reduce the effects of this complexity, was to stabilize the wrist and thumb in the functional position, allowing the grasping of objects with greater effectiveness. This configuration provided benefits in relation to the simplification of the mechanical structure, leaving it compact and light weight. Clinical tests have proven the successfulness of this solution, allowing patients to efficiently perform flexion and extension movements, in addition to holding various objects.

In addition to the complexity of the anatomical and anthropometric characteristics of the human upper limb, this study shows the need to perform tests, observe and accommodate the characteristics of the target audience in question [29]. When performing the elbow movement, it was observed that the synergistic motor behavior of post-stroke individuals altered the positioning and alignment of the device's structure and, consequently, its functioning, requiring changes in its structure.

This study corroborates with Gull et al. [29] that the Exoskeleton design should be kinematically compatible with the variable anthropomorphic parameters of the end-user. The incorrect alignment between exoskeleton and the human upper limb makes patients uncomfortable when they are given therapy. Thus, it is observed that the proper alignment between the exoskeleton and the user's anatomical joints is still a challenge that needs to be overcome to achieve effectiveness with the use of the device [28,32]. 


\section{Conclusions}

This work presented a new robotic device composed of robust motors and actuators, which are located outside the device structure. Despite not being portable for daily use, the device was able to perform the movements effectively, being possible to use it exclusively in rehabilitation clinics, in a way that helps in the recovery of the upper limb. Future studies are necessary to improve the mechanical structure of the orthosis, making it simpler, lighter, and portable, making it possible to use it at home. Besides, it is important to assess its effect on the level of rehabilitation in individuals with upper limb impairment.

Acknowledgments: The authors would like to thank the Universidade Federal de Minas Gerais, Pontifícia Universidade Católica de Minas Gerais, and the Graduate Program in Mechanical Engineering for the support available to carry out this project. We also thank our funders which were: Financiadora de Estudos e Projetos (FINEP: 01.12.0476.00), Fundação de Amparo à Pesquisa do Estado de Minas Gerais (FAPEMIG) and Coordenação de Aperfeiçoamento de Pessoal de Nível Superior (Capes): finance code 001.

Author Contributions: F. M. R. M. F and G.P.R. conceptualization, data curation and writing and editing; L.G.T. and F.H.L.B. data curation and writing; N.B.C.A.; A.M.M.; J.P.F.B.; T.G.S. and R.M.A.D. data analysis, writing and editing; A.M.V.N.V.P. Supervision; C.B.S.V. Supervision, funding acquisition and project administration.

Conflicts of Interest: The authors declare no conflict of interest.

\section{Abbreviations}

The following abbreviations are used in this manuscript:

DC: Direct Current

UE: Upper Extremity

RT: Robot-assisted Therapy

PLA: Lactic polyacid

PWM: Pulse Width Modulation

CG: Center of gravity

To: Torque output

ICF: Free and Informed Consent Form

COEP: Research Ethics Committee

VAS: Visual Analogue Scale

UFMG: Federal University of Minas Gerais

LABBIO: Bioengineering Laboratory

ICR: Instantaneous centers of rotation

\section{References}

1. ROYAL COLLEGE OF PHYSICIANS National clinical guidelines for stroke; 5 th ed.; Intercollegiate Stroke Working: Londres, 2016;

2. Feigin, V.L.; Abajobir, A.A.; Abate, K.H.; Abd-Allah, F.; Abdulle, A.M.; Abera, S.F.; Abyu, G.Y.; Ahmed, M.B.; Aichour, A.N.; Aichour, I.; et al. Global, regional, and national burden of neurological disorders during 1990-2015: a systematic analysis for the Global Burden of Disease Study 2015. Lancet Neurol. 2017, 16, 877-897, doi:10.1016/S1474-4422(17)30299-5.

3. Katan, M.; Luft, A. Global Burden of Stroke. Semin. Neurol. 2018, 38, 208-211, doi:10.1055/s-0038-1649503.

4. SVEEN, U.; BAUTZ-HOLTER, E.; MARGRETHE SODRING, K.; BRUUN WYLLER, T.; LAAKE, K. Association between impairments, self-care ability and social activities 1 year after stroke. Disabil. Rehabil. 1999, 21, 372-377, doi:10.1080/096382899297477.

5. Maciejasz, P.; Eschweiler, J.; Gerlach-Hahn, K.; Jansen-Troy, A.; Leonhardt, S. A survey on robotic devices for upper limb rehabilitation. J. Neuroeng. Rehabil. 2014, 11, 3, doi:10.1186/1743-0003-11-3.

6. Nakayama, H.; Stig Jørgensen, H.; Otto Raaschou, H.; Skyhøj Olsen, T. Recovery of upper extremity function in stroke patients: The Copenhagen stroke study. Arch. Phys. Med. Rehabil. 1994, 75, 394-398, doi:10.1016/0003-9993(94)90161-9. 
7. Kwakkel, G.; Kollen, B.J.; Krebs, H.I. Effects of Robot-Assisted Therapy on Upper Limb Recovery After Stroke: A Systematic Review. Neurorehabil. Neural Repair 2008, 22, 111-121, doi:10.1177/1545968307305457.

8. Galea, M.; Khan, F.; Amatya, B.; Elmalik, A.; Klaic, M.; Abbott, G. Implementation of a technology-assisted programme to intensify upper limb rehabilitation in neurologically impaired participants: A prospective study. J. Rehabil. Med. 2016, 48, 522-528, doi:10.2340/16501977-2087.

9. Huang, V.S.; Krakauer, J.W. Robotic neurorehabilitation: a computational motor learning perspective. J. Neuroeng. Rehabil. 2009, 6, 5, doi:10.1186/1743-0003-6-5.

10. Brewer, B.R.; McDowell, S.K.; Worthen-Chaudhari, L.C. Poststroke Upper Extremity Rehabilitation: A Review of Robotic Systems and Clinical Results. Top. Stroke Rehabil. 2007, 14, 22-44, doi:10.1310/tsr1406-22.

11. Sivan, M.; O'Connor, R.; Makower, S.; Levesley, M.; Bhakta, B. Systematic review of outcome measures used in the evaluation of robot-assisted upper limb exercise in stroke. J. Rehabil. Med. 2011, 43, 181-189, doi:10.2340/16501977-0674.

12. Ferreira, F.M.R.M.; Chaves, M.E.A.; Oliveira, V.C.; Van Petten, A.M.V.N.; Vimieiro, C.B.S. Effectiveness of robot therapy on body function and structure in people with limited upper limb function: A systematic review and meta-analysis. PLoS One 2018, 13, e0200330, doi:10.1371/journal.pone.0200330.

13. Duret, C.; Grosmaire, A.-G.; Krebs, H.I. Robot-Assisted Therapy in Upper Extremity Hemiparesis: Overview of an Evidence-Based Approach. Front. Neurol. 2019, 10, doi:10.3389/fneur.2019.00412.

14. Prange, G.B.; Jannink, M.J.A.; Groothuis-Oudshoorn, C.G.M.; Hermens, H.J.; IJzerman, M.J. Systematic review of the effect of robot-aided therapy on recovery of the hemiparetic arm after stroke. J. Rehabil. Res. Dev. 2006, 43, 171, doi:10.1682/JRRD.2005.04.0076.

15. Norouzi-Gheidari, N.; Archambault, P.S.; Fung, J. Effects of robot-assisted therapy on stroke rehabilitation in upper limbs: Systematic review and meta-analysis of the literature. J. Rehabil. Res. Dev. 2012, 49, 479, doi:10.1682/JRRD.2010.10.0210.

16. Mehrholz, J.; Pohl, M.; Platz, T.; Kugler, J.; Elsner, B. Electromechanical and robot-assisted arm training for improving activities of daily living, arm function, and arm muscle strength after stroke. Cochrane Database Syst. Rev. 2015, doi:10.1002/14651858.CD006876.pub4.

17. Veerbeek, J.M.; Langbroek-Amersfoort, A.C.; van Wegen, E.E.H.; Meskers, C.G.M.; Kwakkel, G. Effects of Robot-Assisted Therapy for the Upper Limb After Stroke. Neurorehabil. Neural Repair 2017, 31, 107-121, doi:10.1177/1545968316666957.

18. Zhang, K.; Chen, X.; Liu, F.; Tang, H.; Wang, J.; Wen, W. System Framework of Robotics in Upper Limb Rehabilitation on Poststroke Motor Recovery. Behav. Neurol. 2018, 2018, 1-14, doi:10.1155/2018/6737056.

19. Bertani, R.; Melegari, C.; De Cola, M.C.; Bramanti, A.; Bramanti, P.; Calabrò, R.S. Effects of robot-assisted upper limb rehabilitation in stroke patients: a systematic review with meta-analysis. Neurol. Sci. 2017, 38, 1561-1569, doi:10.1007/s10072-017-2995-5.

20. Mehrholz, J.; Pohl, M.; Platz, T.; Kugler, J.; Elsner, B. Electromechanical and robot-assisted arm training for improving activities of daily living, arm function, and arm muscle strength after stroke. Cochrane Database Syst. Rev. 2018, doi:10.1002/14651858.CD006876.pub5.

21. Rúbio, G. de P.; Martins Ferreira, F.M.R.; Brandão, F.H. de L.; Machado, V.F.; Tonelli, L.G.; Martins, J.S.R.; Kozan, R.F.; Vimieiro, C.B.S. Evaluation of Commercial Ropes Applied as Artificial Tendons in Robotic Rehabilitation Orthoses. Appl. Sci. 2020, 10, 920, doi:10.3390/app10030920.

22. Rúbio, G. de P.; Ferreira, F.; de Lisboa Brandão, F.H.; Machado, V.; Tonelli, L.; Kozan, R.F.; Vimieiro, C. Design of Actuators Applied to a Upper Limb Orthosis. In Proceedings of the Proceedings of the 25th International Congress of Mechanical Engineering; ABCM, 2019.

23. Tittiranonda, P.; Rempel, D.; Armstrong, T.; Burastero, S. Workplace Use of an Adjustable Keyboard: Adjustment Preferences and Effect on Wrist Posture. Am. Ind. Hyg. Assoc. J. 1999, 60, 340-348, doi:10.1080/00028899908984451.

24. Wechsler, L.R.; Bates, D.; Stroemer, P.; Andrews-Zwilling, Y.S.; Aizman, I. Cell Therapy for Chronic Stroke. Stroke 2018, 49, 1066-1074, doi:10.1161/STROKEAHA.117.018290.

25. Bohannon, R.W.; Smith, M.B. Interrater Reliability of a Modified Ashworth Scale of Muscle Spasticity. Phys. Ther. 1987, 67, 206-207, doi:10.1093/ptj/67.2.206.

26. Fugl-Meyer, A.R.; Jääskö, L.; Leyman, I.; Olsson, S.; Steglind, S. The post-stroke hemiplegic patient. 1. a method for evaluation of physical performance. Scand. J. Rehabil. Med. 1975, 7, 13-31.

27. Bertolucci, P.H.F.; Brucki, S.M.D.; Campacci, S.R.; Juliano, Y. O Mini-Exame do Estado Mental em uma população geral: impacto da escolaridade. Arq. Neuropsiquiatr. 1994, 52, 01-07, doi:10.1590/S0004282X1994000100001. 
28. Islam, M.R.; Spiewak, C.; Rahman, M.H.; Fareh, R. A Brief Review on Robotic Exoskeletons for Upper Extremity Rehabilitation to Find the Gap between Research Porotype and Commercial Type. Adv. Robot. Autom. 2017, 06, doi:10.4172/2168-9695.1000177.

29. Gull, M.A.; Bai, S.; Bak, T. A Review on Design of Upper Limb Exoskeletons. Robotics 2020, 9, 16, doi:10.3390/robotics9010016.

30. Silveira Romero, R.C. da; Machado, A.A.; Costa, K.A.; Reis, P.H.R.G.; Paiva Brito, P.; Santos Vimieiro, C.B. Development of a Passive Prosthetic Hand That Restores Finger Movements Made by Additive Manufacturing. Appl. Sci. 2020, 10, 4148, doi:10.3390/app10124148.

31. Neumann, D.A. CINESIOLOGIA DO APARELHO MUSCULOESQUELÉTICO; 2a .; Elsevier: Rio de Janeiro, 2011; ISBN 978-85-352-3966-9.

32. Esmaeili, M.; Gamage, K.; Tan, E.; Campolo, D. Ergonomic considerations for anthropomorphic wrist exoskeletons: A simulation study on the effects of joint misalignment. In Proceedings of the 2011 IEEE/RSJ International Conference on Intelligent Robots and Systems; IEEE, 2011; pp. 4905-4910.

(C) 2020 by the authors; licensee MDPI, Basel, Switzerland. This article is an open access article distributed under the terms and conditions of the Creative Commons by Attribution (CC-BY) license (http://creativecommons.org/licenses/by/4.0/). 